\title{
Cutaneous ulcers in association with sprue-like enteropathy secondary to losartan
}

\author{
Francis Essien ${ }^{1}$, Wassem Juakiem ${ }^{1}$, Joshua Tate ${ }^{1}$, and Jared Roberts ${ }^{1}$ \\ ${ }^{1}$ Keesler Air Force Base
}

May 18, 2021

\begin{abstract}
Angiotensin receptor antagonist associated enteropathy is becoming increasingly prevalent within the literature. We report a case of sprue-like enteropathy associated with Losartan with a unique cutaneous manifestation. Our case of Losartan associated enteropathy further suggests that sprue-like disease may be a class effect of ARBs.
\end{abstract}

\section{Introduction:}

Angiotensin receptor blocker (ARB) induced sprue-like enteropathy is a rare adverse effect of this class of medication but is increasingly recognized as a potential side effect that clinicians should be aware. The first published cases were from the Mayo Clinic in a case series of Olmesartan induced sprue-like enteropathy. While reported clinical manifestations of these patients included abdominal pain, severe weight loss, chronic diarrhea, and severe electrolyte derangements, there was no description of cutaneous manifestations. Since that time, only one case of ARB induced sprue-like enteropathy with cutaneous manifestations has been noted and was associated with Olmesartan, specifically. Symptoms in the above cases resolved within 3-12 months of medication discontinuation. Currently sprue-like enteropathy has yet to be classified as class effect among ARBs; however, there are several cases within the literature that advocate in favor of this idea. We report a case of sprue-like enteropathy associated with losartan with a unique cutaneous manifestation.

\section{Case Report:}

A 59-year-old female had an abrupt presentation of diarrhea, abdominal discomfort, weight loss and several large spontaneous ulcerations on the abdomen, limited within scar tissue from a remote bowel perforation surgery (Figure 1a). The combination of diarrhea and ulcerations was concerning for inflammatory bowel disease, pyoderma gangrenosum, or malignancy. Laboratory investigations demonstrated anemia and electrolyte derangements to include hyponatremia, hypocalcemia, hypokalemia, and hypomagnesemia. Chronic diarrhea work-up was negative, but lactoferrin and calprotectin were elevated (Table 1). A punch biopsy at the edge of an ulcer showed extensive dermal fibrosis with chronic inflammation, numerous eosinophils and granulation tissue (Figure $1 \mathrm{bc}$ ). PAS and Lu-5 cytokeratin stains were performed and were unremarkable. The lack of acute or granulomatous inflammation made infection less likely. Otherwise, the classical features of pyoderma gangrenosum, infection, and malignancy were not appreciated. Endoscopy was notable for duodenal villous atrophy, edematous mucosa with scattered area of loss of vascular pattern and ulceration in the proximal colon but histology demonstrated benign colonic mucosa. After months of otherwise unremarkable exhaustive evaluation there was no clear underlying cause. Of note, the patient had been treated with losartan for more than 10-years for hypertension and given the reports of ARB induced sprue-like enteropathy, losartan was discontinued in October 2019. On follow up one month after medication was discontinued patient noted improvement of the diarrhea and the wounds started healing. Patient 
followed up with wound care and over the next several months the wounds completely resolved as well as her gastrointestinal symptoms. (Figure 1c).

\section{Discussion:}

The increasing armamentarium of pharmacological agents has resulted in a multifactorial fusillade of effects within the gastrointestinal and cutaneous systems. The small intestine is the most common site of adverse drug reaction due to the delicate balance between the enteric and central nervous system ${ }^{1}$. Often there is a temporal relationship between the drug ingestion and onset of symptoms, but this can sometimes manifest inconsistently and unpredictably ${ }^{1,2}$. Very few cases of ARB induced sprue-like enteropathy, other than olmesartan, have been reported. There has only been one reported case with cutaneous manifestations and ARB induced sprue-like enteropathy. There have been several case reports and cohort studies over the last decade which have demonstrated a sprue-like enteropathy presenting with abdominal pain, chronic diarrhea, and weight loss in association with ARB use ${ }^{1,2}$. In 2016, Hammoudi et al published the first case of olmesartan induced enteropathy with 'papulo-erythematous lesions and scabs' with full resolution within a week following cessation of the medication ${ }^{3}$. The pathophysiology of ARB induced sprue-like enteropathy is currently unknown with even less understood regarding the connection to a cutaneous manifestation. However, the punch biopsy and duodenal samples did demonstrate findings consistent with drug eruption, and after months of symptoms, a few hospitalizations, and several failed therapies, the treatment that ended up improving her condition was discontinuation of the losartan.

Several studies have demonstrated that many of the common pathogenic pathways seen in celiac disease are also present within that of ARB induced sprue-like enteropathy. There is an increased number of CD8+ positive T cells, and granzyme positive B cells resulting in destruction of the lamina propria and epithelial layer ${ }^{2,5,6}$. Previous studies have demonstrated an increased number of CD8+ positive T cells which express interleukin 15 and $15 \mathrm{R}$ resulting in the disruption of tight junction complexes such as in refractory celiac sprue $^{1}$. Given some of the known histopathology overlap observed in celiac disease and ARB induced spruelike enteropathy, a cutaneous manifestation is not unexpected, although the cutaneous manifestations may differ. Celiac disease is associated with dermatitis herpetiformis whereas ARB induced sprue enteropathy may cause ulcerative lesions ${ }^{5,6}$. Our case of losartan induced enteropathy further suggests that sprue-like enteropathy with cutaneous manifestations may be a unique but rare class effect of ARBs and should be considered when evaluating patients with similar clinical findings.

\section{References:}

1. Scarpignato, C., Bjarnason, I. Drug-Induced Small Bowel Injury: a Challenging and Often Forgotten Clinical Condition. Current Gastroenterology Reports. 2019; 21: 55

2. Marietta et al. Immunopathogenesis of Olmesartan-Associated Enteropathy. Aliment Pharmacology Therapy. 2015;42:1303-1314

3. Hammoudi, N., Dior, M., Giraud, V., Coffin, B. Olmesartan-induced Enteropathy, associated with cutaneous lesions. Clinical Case Reports. 2016;4:379-382

4. Bolognia, J., Jorizzo, J., Schaffer, J., Dermatology fourth edition. Elsevier Saunders, 2012.

5. Reunala T, Salmi TT, Hervonen K, Kaukinen K, Collin P. Dermatitis Herpetiformis: A Common Extraintestinal Manifestation of Coeliac Disease. Nutrients. 2018;10:602.

6. Melis, C., Struyve, M., Steelandt, T., Neuville, B., Deraedt, K. Sprue-like enteropathy, do not forget olmesartan! Digestive and Liver Disease. 2018;50:621-624

The authors confirm contribution to the paper as follows:

Study conception and design: Francis Essien D.O., Wassem Juakiem M.D., Jared Roberts M.D.

Data collection: Francis Essien D.O.

Analysis and interpretation of results: Francis Essien D.O., Jared Roberts M.D. 
Draft manuscript preparation: Francis Essien D.O., Joshua Tate M.D.

Figure 1. A. $9 \mathrm{~cm} \times 9 \mathrm{~cm}$ scar with multiple ulcerated lesions on the anterior supra-umbilical region)

B. Histopathology: ulceration with extensive dermal fibrosis, chronic inflammation, numerous eosinophils and granulation tissue

C. 1 month following losartan discontinuation.

\section{Hosted file}

Figures.pdf available at https://authorea.com/users/405924/articles/522484-cutaneous-ulcersin-association-with-sprue-like-enteropathy-secondary-to-losartan 